\title{
Detecting antibody-labeled BCG MNPs using a magnetoresistive biosensor and magnetic labeling technique
}

\author{
Teresa R. G. Barroso ${ }^{1,2,3, a *}$, Verónica C. Martins ${ }^{3, b}$, Filipe Cardoso ${ }^{4}$, \\ Susana Cardoso ${ }^{4}$, Jorge Pedrosa ${ }^{1,2}$, Margarida Correia-Neves ${ }^{1,2}$, José Rivas ${ }^{3}$ \\ and Paulo P. Freitas ${ }^{3, c}$
}

\author{
${ }^{1}$ ICVS: Life and Health Sciences Research Institute, School of Health Sciences, University of \\ Minho, Campus de Gualtar, 4710-057 Braga, Portugal \\ 2 ICVS/3B's - PT: Government Associate Laboratory, Braga/ Guimarães, Campus de Gualtar, \\ 4710-057 Braga, Portugal \\ 3 INL: International Iberian Nanotechnology Laboratory, Avenida Mestre José Veiga s/n, 4715-330 \\ Braga, Portugal \\ 4 INESC-MN: Instituto de Engenharia de Sistemas e Computadores - Microsistemas e \\ Nanotecnologias, Rua Alves Redol, 9, 1000-029 Lisbon, Portugal \\ ateresa.barroso@gmail.com, ${ }^{b}$ veronica.romao@inl.int, ${ }^{c}$ paulo.freitas@inl.int
}

Keywords: Tuberculosis, nanotechnology, diagnosis, sandwich-immunoassay, magnetic nanoparticles, magnetoresistive biochip

\begin{abstract}
Tuberculosis is still a major global health concern, causing the estimated death of 1.5 million people per year and being associated with high morbidity. The development of point-of-care diagnostic tools for tuberculosis is mandatory, especially because the fast and accurate detection of the slow-growing Mycobacterium tuberculosis by the conventional diagnostic tests is difficult.

The objective of this work was to develop the first steps to achieve a portable method for the diagnosis of tuberculosis, by a sandwich-immunoassay combined with magnetoresistive biochip technology.

With the purpose of conjugating $250 \mathrm{~nm}$ streptavidin-coated magnetic nanoparticles with anti- $M$. tuberculosis biotinylated antibodies, Mycobacterium bovis Bacillus Calmette-Guérin was used as a surrogate for $M$. tuberculosis bacteria. After magnetic capture, target bacteria were brought in contact with the surface of the magnetoresistive biochip previously functionalized with a secondary anti-M. tuberculosis antibody. Magnetically labeled cells were detected by an array of spin-valve sensors, which change their electrical resistance in the presence of the fringe field of the magnetic particles. Optimization studies on the efficiency of the magnetic capture and further recognition of the bacteria by the secondary antibody on the biochip surface were conducted. The results on the magnetoresistive biochip showed a clear difference in the signal between specific and control (nonspecific) sensors, suggesting the usefulness of this technique as a potential biorecognition tool for the development of a point-of-care diagnostic method for tuberculosis.
\end{abstract}

\section{Introduction}

Mycobacterium tuberculosis is an old threat that causes one of the main health problems worldwide. In 2013, the World Health Organization (WHO) appraised tuberculosis (TB) as the second leading cause of mortality from a single infectious agent, after the human immunodeficiency virus (HIV), causing the death of 1.5 million people, with 360000 cases among HIV-positive patients. Moreover, TB is a destructive disease associated with high morbidity (estimation of 9 million people in 2013, including 1.1 million HIV-positive patients), with $80 \%$ of the cases in poor resources countries (22 high-burden countries) [1].

Accurate early diagnosis and correct treatment would deeply contribute to the control of TB globally, but the diagnosis is challenging. On one hand, sputum microscopy, although rapid, simple 
and inexpensive, has low sensitivity, and, on the other hand, the gold-standard culture method has limitations of speed (up to 8 weeks). Extensive experimental work has been developed over the last decades and current research on alternative TB diagnostic platforms aims at the development of point-of-care tools to replace culture-based TB detection tests [1,2]. The objective is achieving rapid, accurate, robust, inexpensive and simple tests, based on the direct examination of clinical samples and applicable in conditions of limited resources [3]. Presently, a fast molecular test named Xpert ${ }^{\circledR} M T B / R I F$ has been approved, implemented and reviewed by the WHO. This test has sensitivity comparable to the solid culture method, and detects pulmonary TB as well as rifampicin resistance. Examples of other recent alternative methods researched for the diagnosis of TB include: volatile organic compounds, LATE-PCR, MDR-XDR TB color test, TB rapid screen, TB LAMP, EasyNAT, TB-LAM and immunoassays [2].

Among these techniques aiming to improve the diagnosis of $\mathrm{TB}$, immunoassays of antigen detection tests present the potential advantages of providing rapid and reliable diagnosis with the possibility of correlation with mycobacterial loads [4].

Magnetoresistive (MR) biochips can be used to perform immunoassays, in analogy with the classical enzyme linked immunosorbent assay (ELISA), namely to detect microorganisms such as bacteria. As exemplified in Fig. 1, in a MR biochip, the enzymatic label of the ELISA assay is replaced by a magnetic nanoparticle. MR bioassays can be constructed in the format of a sandwich assays, with probe antibody (Ab), target cell, secondary $\mathrm{Ab}$ and magnetic nanoparticle (MNP). Therefore, the reaction is based on the use of magnetically labeled antibodies (Abs) to attach the bacteria, which are specifically recognized on the biochip surface by a secondary Ab and subsequently detected on MR sensors [5]. The molecular interaction between MNP and pathogens is monitored in real-time, through the variation of the electrical resistance of the sensor, with the magnetic stray fields created by the magnetic labels $[6,7]$.

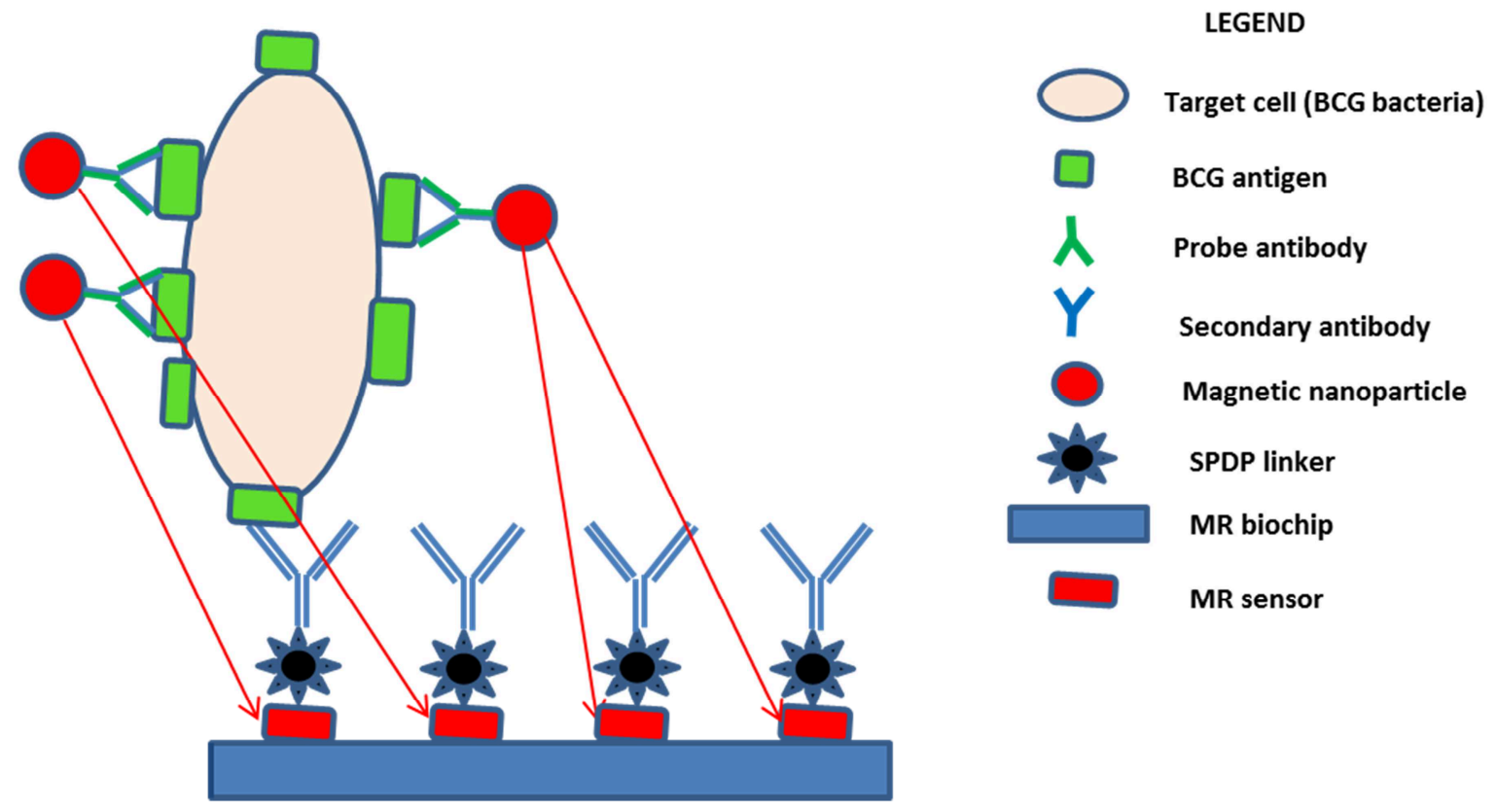

Figure 1. Schematic exemplification of the ELISA sandwich assay that occurs in the magnetoresistive biochip used in the experimental work.

In fact, MR biochips appear as a new solution that has the potential of providing sensitivity, miniaturization, scalability and portability. These platforms could be an alternative to the currently available Xpert®MTB/RIF test, particularly by increasing the sensitivity of the diagnosis. MR sensors have been used to integrate biorecognition assays using probe biomolecules (e.g. Ab, oligonucleotides, bacteriophages, among others) marked with MNP for the detection of biological samples $[6,8]$. 
The herein described novel diagnostic system profits from the conjugation of Abs with MNP, which can be manipulated and precisely detected by MR sensors. The main endeavor of the developed MR platform is that it has the potential to solve the problems of low sensitivity associated with Ab-based diagnosis by reducing the bacterial amount required for the detection, as one sensor can potentially detect a single labelled bacteria. The subsequent work therefore describes the development of a biomolecular detection system of Bacillus Calmette-Guérin (BCG) as a surrogate for Mycobacterium tuberculosis (Mtb) based on MR technology and using specific Abs to the target mycobacteria.

\section{Materials and methods}

2.1. Reagents. All reagents were of analytical grade and water was ultrapure. The reagents for buffer preparation were acquired from Sigma: phosphate buffer (PB; $100 \mathrm{mM} \mathrm{NaH}{ }_{2} \mathrm{PO} 4,100 \mathrm{mM}$ $\mathrm{Na}_{2} \mathrm{HPO}_{4}$, pH 7.4), PB-Tween (PBT; PB with $0.02 \%$ (v/v) of Tween 20), TE buffer (10 mM Tris$\mathrm{HCl}, 1 \mathrm{mM}$ EDTA, pH 7.4), and Bovine serum albumin (BSA, 1\% (w/v) in TE). Sulfo-LC-SPDP (sulfosuccinimidyl6-[3'-(2-pyridyldithio)-propionamido] hexanoate) was purchased from Pierce. Magnetic nanoparticles (nanomag®-D) are produced by Micromod (www.micromod.de) and according to specifications are prepared via the core-shell method with a core of magnetite and a dextran shell, consist of $75-80 \%(\mathrm{w} / \mathrm{w})$ magnetite in a matrix of dextran $(40.000 \mathrm{Da})$. These particles are available with already covalently bound proteins (e.g. streptavidin and protein A). The concentration of the $250 \mathrm{~nm}$ streptavidin modified MNP was $4.9 \times 10^{11}$ particles $/ \mathrm{mL}$.

2.2. Bacterial strain and polyclonal antibodies. Mycobacterium bovis Bacillus CalmetteGuérin Pasteur from the Trudeau Institute Culture Collection (BCG), an attenuated strain of $M$. bovis used as a vaccine against TB was used as a surrogate for MTb, as these bacteria can be manipulated without the need of Biosafety Level 3 facilities. The samples of BCG used were frozen at $-70^{\circ} \mathrm{C}$ after being grown to mid- $\log$ phase in Proskauer-Beck medium with $0.05 \%$ Tween 80 [9]. The bacteria were then cultured at $37^{\circ} \mathrm{C}$ in Difco Middlebrook $7 \mathrm{H} 9$ Broth followed by Difco Mycobacteria 7H11 Agar supplemented with 10\% OADC (oleic acid, albumin, dextrose and catalase). A loopful of BCG was resuspended in PB solution and treated as described by Bettencourt et. al [10] to obtain an optical density of 0.1 at $600 \mathrm{~nm}\left(\mathrm{OD}_{600}\right)$, which corresponds to approximately $1 \times 10^{7}$ bacteria per mL.

The polyclonal antibodies (PAbs) anti - M. tuberculosis (anti-Mtb, PA1-7260, 4-5 mg/mL), biotin conjugated anti - M. tuberculosis (anti-Mtb bio, PA1-73136, 4-5 mg/mL) and antiSalmonella PAbs (anti-Salm, PA1-20811, $1 \mathrm{mg} / \mathrm{mL}$ ), were purchased from Thermo Scientific. The purification of the PAbs was achieved by filtering with centrifugal filters from Millipore (100 kDa cut-off, $10.000 \mathrm{rpm}, 15 \mathrm{~min}$ ) and exchanging to PB buffer to the desired concentration.

2.3 Conjugation of MNP with polyclonal antibodies and BCG. For the conjugation process, $10 \mu \mathrm{L}$ of MNP from the stock solution ( $4.9 \times 10^{9}$ particles) were added to $40 \mu \mathrm{L}$ of PB and placed in a magnetic concentrator (Dynal-biotech) to remove the supernatant and this process was repeated 3 times to wash the MNP from their preservative solution. Different concentrations of the anti-Mtb bio (from 0 to $100 \mu \mathrm{g} / \mathrm{mL}$ in a total volume of $100 \mu \mathrm{L}$ ) were allowed to react with the MNP and the solution was incubated $1 \mathrm{~h}$, at $200 \mathrm{rpm}$ and room temperature (RT). After the PAbs immobilization on the MNP, free reactive sites on the surface of the MNP were blocked with BSA in TE for 30 min, at $200 \mathrm{rpm}$ and RT and washed 3 times with PB using the magnetic concentrator. BSA acts as a blocking agent to avoid non-specific adsorption of other proteins or false positive results. MNP@PAbs in a final concentration of $10^{6} \mathrm{NP} / \mu \mathrm{L}$ were kept at $4{ }^{\circ} \mathrm{C}$ until further use.

The amount of Abs immobilized on the MNP was indirectly estimated by the Bradford Method (Commassie Plus, Pierce, Thermo Scientific) [11]. Several concentrations of the anti-Mtb bio solution were prepared and incubated with the MNP. The PAbs concentration in the supernatant and 
washing solutions after the immobilization was measured at $595 \mathrm{~nm}$ and subtracted to the PAbs initial concentration.

The magnetic capture of the BCG was performed by incubating $1 \mathrm{~mL}$ of $5 \times 10^{6}$ cells $/ \mathrm{mL}$ with different concentrations of the anti-Mtb bio previously functionalized with MNP $\left(10^{6} \mathrm{NP} / \mu \mathrm{L}\right)$ for $1 \mathrm{~h}$, at $200 \mathrm{rpm}$ and RT. Then, the cells were washed 3 times with PB using the magnetic concentrator and ressuspended in water. A Kinyoun acid-fast staining was used to simplify the cell counting in a Petroff-Hausser chamber and determine the BCG cell capture efficiency by the functionalized MNP [12]. The number of captured bacteria was calculated by subtracting the supernatant and washing solutions from the magnetic capturing procedure to the original cell suspension.

2.4 Biomolecular recognition of the conjugated MNP on gold substrates. $\mathrm{Si} / 5 \mathrm{~mm} \mathrm{Cr} / 40 \mathrm{~nm}$ $\mathrm{Au}$ substrates were used to test the immunodetection efficiency of magnetically captured BCG (MNP@PAbs@BCG) by immobilized Abs on the gold surface. After copious cleaning of the Au surface, a droplet of $20 \mu \mathrm{L}$ of sulfo-LC-SPDP at $1 \mathrm{mg} / \mathrm{mL}$ in PB was placed over the entire surface of the substrates for $1 \mathrm{~h}$, at RT [8]. Sulfo-LC-SPDP is a bifunctional crosslinker that establishes the binding between gold surfaces and functional amino groups of Abs molecules. The substrates were then rinsed with PB and ultrapure water and gently dried with a nitrogen gun. Then, 4 droplets of 2 $\mu \mathrm{L}$ of both positive (anti-Mtb) and negative (anti-Salm) control PAbs, in a concentration of 50 $\mu \mathrm{g} / \mathrm{mL}$, were manually spotted in the gold surface and incubated for $2 \mathrm{~h}$ at RT, in a humidified atmosphere. The gold substrate was washed in PB to remove unbound PAbs and gently dried with a nitrogen gun. To block nonspecific adsorption between cells and MNP, $20 \mu \mathrm{L}$ of 1\% BSA in TE were put over the surface and incubated $1 \mathrm{~h}$, at RT, in a humidified atmosphere. The substrate was washed twice with TE buffer to remove the excess of blocking solution and once with PB. Then, 20 $\mu \mathrm{L}$ of MNP@PAbs@BCG were deposited over the gold surface and incubated 1 h, followed by 3 washing steps with moderate agitation: $5 \mathrm{~min}$ in PB, $5 \mathrm{~min}$ in PBT and $5 \mathrm{~min}$ in water. At the end, microscopic images were taken using an optical stereomicroscope (Nikon SMZ 1500) and the image processing software Image $J$ was used to analyze the surface coverage over the gold substrate. This technique involves the determination of the area of the pictures that is covered by MNP, allowing an estimation of the density of MNP@PAbs@BCG immobilized on the PAbs spots.

2.5 Optimization of the biomolecular recognition of MNP@PAbs@BCG on the gold chip. The MR-biochip was produced by INESC MN as described by Martins and colleagues [13]. The biochip surface that detects MNP labels consists of patterned $\mathrm{Cr} / \mathrm{Au}$ pads over $\mathrm{SiN}$, with MR sensors beneath. Basically, the MR chip includes two separate gold sensing areas disposed in two areas, each one with 3 groups of $5 \mathrm{U}$-shaped $2.5 \times 80 \mu \mathrm{m}^{2}$ spin valve sensors.

MNP@PAbs@BCG with an initial concentration of the anti-Mtb bio of $25 \mu \mathrm{g} / \mathrm{mL}, 10^{6} \mathrm{NP} / \mu \mathrm{L}$ of MNP and $5 \times 10^{6}$ cells $/ \mathrm{mL}$ of BCG were used to enhance the specific binding to Au pads in further measurements on the MR-biochip, through experiments with uncapsulated biochips (without the MR measurement system). The BCG cells were captured as described in section 2.4, but after the last washing step, bacteria were ressuspended in 3 different solutions: water, PB or PBT. Magnetically captured bacteria were then biorecognized by PAbs on the surface of the biochip with the same procedure described before for the Au substrates, but a $2 \mu \mathrm{L}$ droplet of the specific antiMtb was deposited on the left pad and the same volume of the non-specific anti-Salm was deposited on the right pad. Finally, optical microscopic images were taken using an inverted microscope (Nikon ECLIPSE L200N) and the image processing software Image $\mathbf{J}$ was used to analyze the surface coverage over the gold pads.

2.6 MR-biochip measurement. The surface of the MR biochip was functionalized as previously described for the gold substrates and in the same concentrations as for the uncapsulated microchips. The left area was used to measure the positive control (anti-Mtb) and the right column for the negative control (anti-Salm). After functionalization, the biochip was introduced on the portable electronic platform produced by INESC [7]. A microfluidic channel connected to a syringe 
controlled by a syringe pump (New Era NE-300) was used to load the MNP@PAbs@BCG solution over the sensitive area of the biochip [14].

The sensors were used under standard measurement condition with a $1 \mathrm{~mA}$ biasing current, while an external $3 \mathrm{mT}$ magnetic bias field and $1.35 \mathrm{mT}$ rms AC was used to magnetize the MNP [7]. The acquisition of the baseline signal was achieved by a $15 \mathrm{~min}$ flow of $\mathrm{PB}$ inside the microfluidic channel. Then, $5 \mu \mathrm{L}$ of the MNP@PAbs@BCG in PB solution were introduced inside the channel and allowed to settle down $30 \mathrm{~min}$ for antigen recognition by the secondary Abs on the surface of the sensor (sandwich-type bioassay). The unbound MNP were washed-out by rinsing with a solution of PBT, firstly at a flow rate of $5 \mu \mathrm{L} / \mathrm{min}$ for $5 \mathrm{~min}$ and then $50 \mu \mathrm{L} / \mathrm{min}$ for $5 \mathrm{~min}$. During this procedure the signal acquisition was sequentially measured for all sensors inside the MR biochip. The binding signal is proportional to the number of magnetically labeled bacteria successfully bound to the surface of the sensor, after the washing steps. The differential signal ( $\Delta \mathrm{V}_{\text {binding }}$ ) is the variation between the baseline signal and the binding signal. In order to be able to compare different sensors in the same assay and among different biochips, the $\Delta \mathrm{V}_{\text {binding }}$ is normalized to each sensor baseline signal $\left(\mathrm{V}_{\text {sensor }}\right)$ at the measurement conditions $\left(\mathrm{DV} / \mathrm{V}_{\text {sensor }}\right)[13]$.

\section{Results and discussion}

3.1 MNP functionalization with PAbs and BCG capture efficiencies. Fig. 2 shows the density of immobilized PAbs in the MNP as a function of the initial concentration of the anti-Mtb bio in solution. Several PAbs concentrations were tested $(0,6.5,12.5,25,50$ and $100 \mu \mathrm{g} / \mathrm{mL})$ and the number of immobilized PAbs per MNP was calculated. The Bradford method [11] allowed an estimation of the maximum number of PAbs, around 2900 per MNP for an initial Ab concentration of $100 \mu \mathrm{g} / \mathrm{mL}$.

It is important to control the density of immobilized PAbs on the surface of MNP for the design and optimization of MR biosensors [5]. Very sparse surface density of PAbs will result in poor capture efficiencies and conversely when the surface density increases, steric hinderance of target recognition starts to appear $[13,15]$.

As showed in Fig. 2, the Ab surface density followed a saturation profile, with maximum surface densities of around $2900 \mathrm{PAbs} / \mathrm{MNP}$. The results confirmed that it was possible to successfully functionalize the MNP with anti-Mtb bio, using the biotin-streptavidin affinity binding. The biotinstreptavidin interaction is known to be very efficient due to the high affinity between these two biomolecules, which allows biotinylated Abs to be strongly conjugated with streptavidin coated MNP [5,16,17]. 


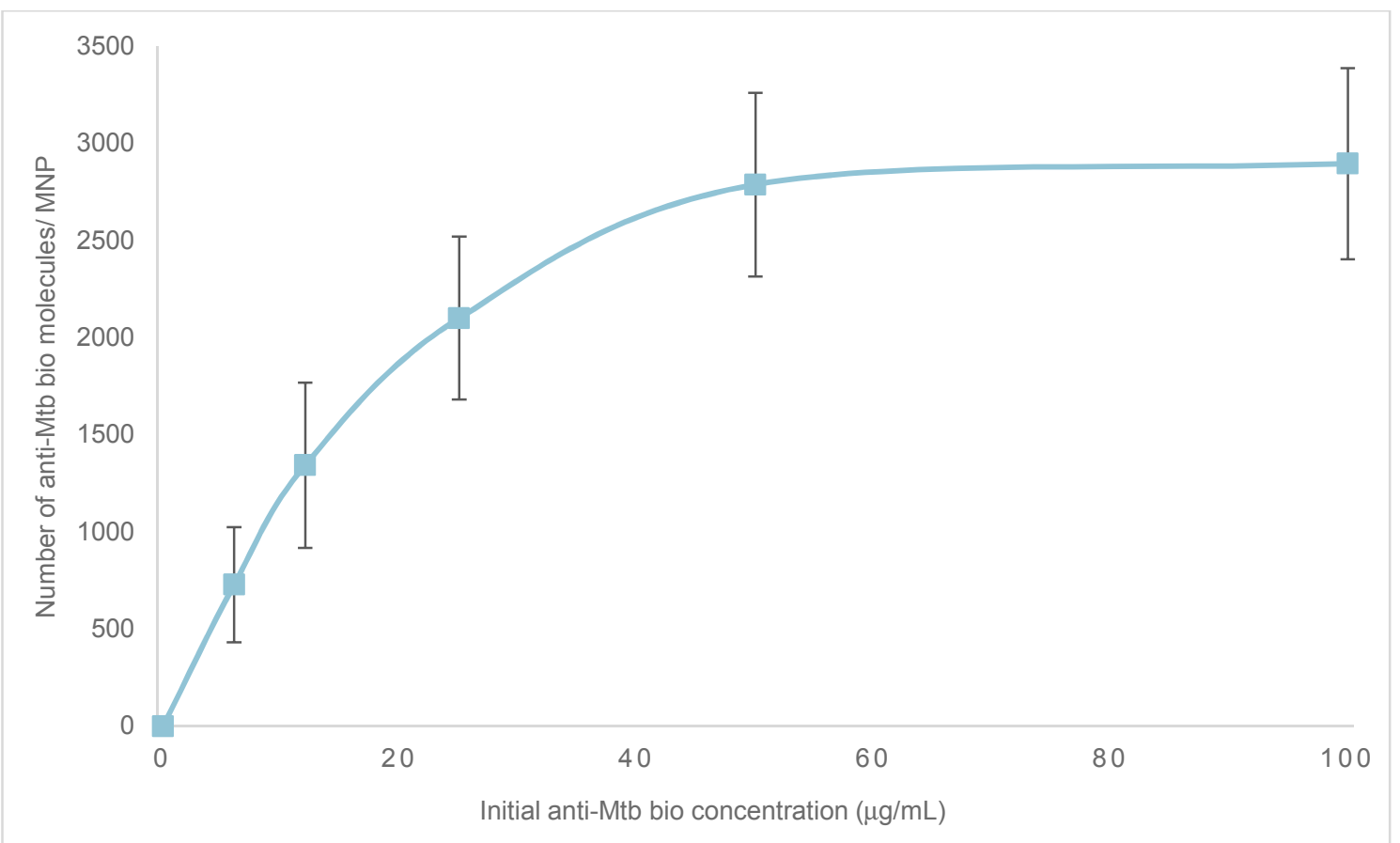

Figure 2. Efficiency of the immobilization between streptavidin modified MNP and the specific biotin conjugated anti-Mtb bio, as a function of the initial concentration of PAbs in solution and estimated by the Bradford Method.

Fig. 3 presents the capture efficiency of BCG varying the initial PAbs concentration in PB solution. We used a solution of BCG + MNP (without anti-Mtb bio) as a negative control. The spatial accessibility of the PAbs is an important factor to ensure the performance of the system, as active sites of the antibodies have to be available to react with the antigen. [5,18].

The concentration of PAbs that maximized the capture efficiency (around 86\%) of BCG cells by bioconjugated MNP was $25 \mu \mathrm{g} / \mathrm{mL}$. Unspecific adsorptions between bacterial cells and MNP explain the result on the negative control. BCG cells were successfully captured and specifically recognized by MNP functionalized with anti-Mtb bio.

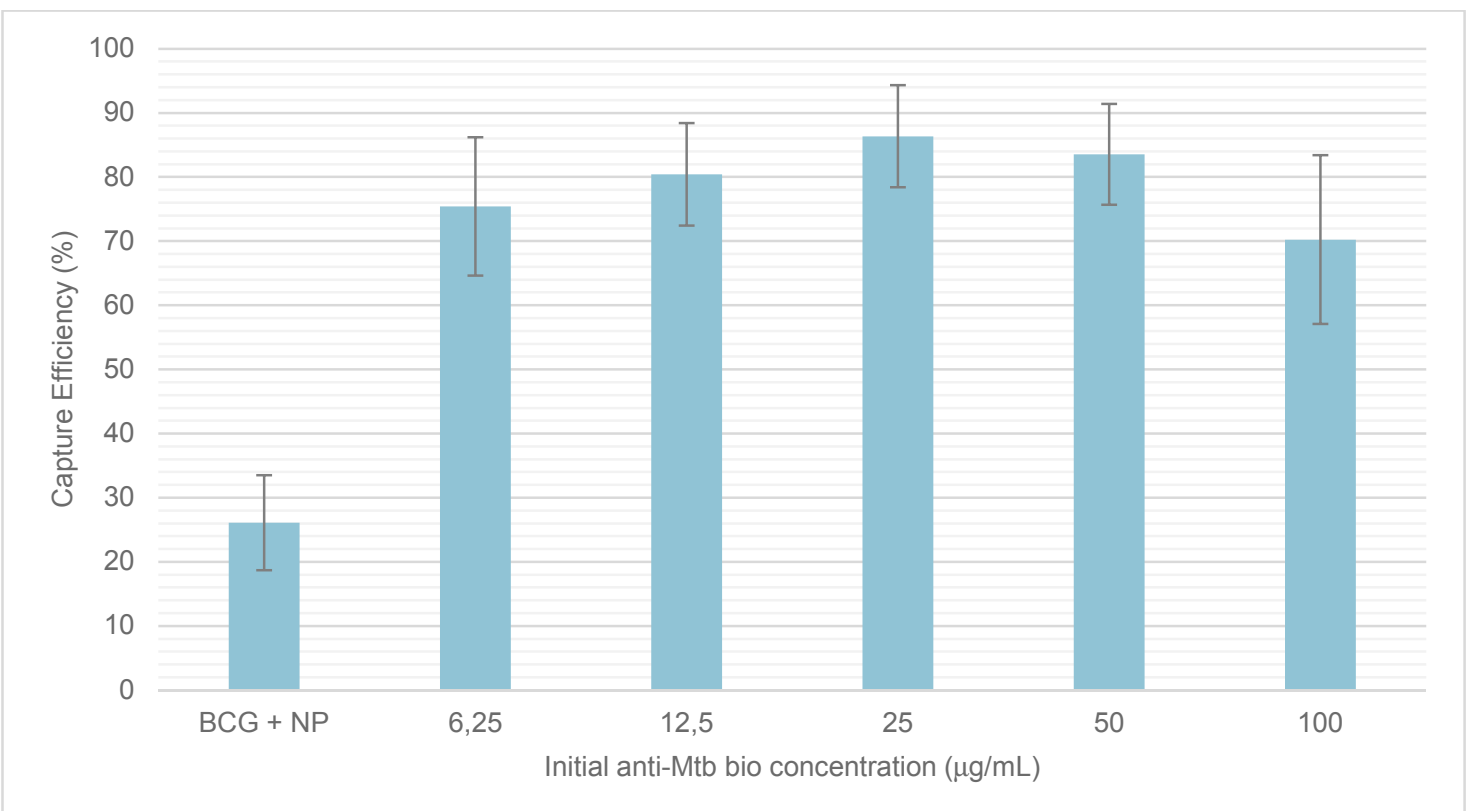

Figure 3. Evaluation of the capture efficiency of BCG cells by the MNP functionalized with the specific anti-Mtb bio, quantified by the Petroff-Hausser counting chamber method. 
3.2 Immobilization of the MNP@PAbs@BCG on gold substrates. The process of immobilization on the surface of the biochip highly influences the performance of the immunoassay [5,18]. Fig. 4 presents the surface coverage on the spot of the secondary anti-Mtb by the MNP@PAbs@BCG, depending on the initial concentration of anti-Mtb bio previously used to functionalize MNP.

Gold substrates mimic the surface of the MR biochip and were observed under a stereoscopic microscope to estimate the MNP@PAbs@BCG immobilization efficiency by the Image J software, which is determined by the surface coverage. The BCG + MNP (without anti-Mtb bio), PAbs + MNP (without BCG) and only BCG (without MNP or anti-Mtb bio) were used as negative controls.

Results showed the effective biorecognition of MNP@PAbs@BCG by secondary nonbiotinylated anti-Mtb on the surface of gold substrates. The concentration of anti-Mtb bio, previously functionalized with MNP, that provided the better surface coverage (around 90\%) was $25 \mu \mathrm{g} / \mathrm{mL}$. Nonspecific adsorptions could explain why negative controls present some degree of immobilization efficiencies even with BSA as a blocking agent in solution. An inverse proportionality between the concentration of anti-Mtb bio and the capture efficiency of MNP@PAbs@BCG on gold substrates was demonstrated from $50 \mu \mathrm{g} / \mathrm{mL}$, which was also observed in the evaluation of the capture efficiency of BCG cells by the Petroff-Hausser Counting Chamber method. This characteristic had also been verified by Martins and coworkers [5], which explained that despite the fact that more Abs can be attached as the concentration increases, the biological functionality becomes compromised.

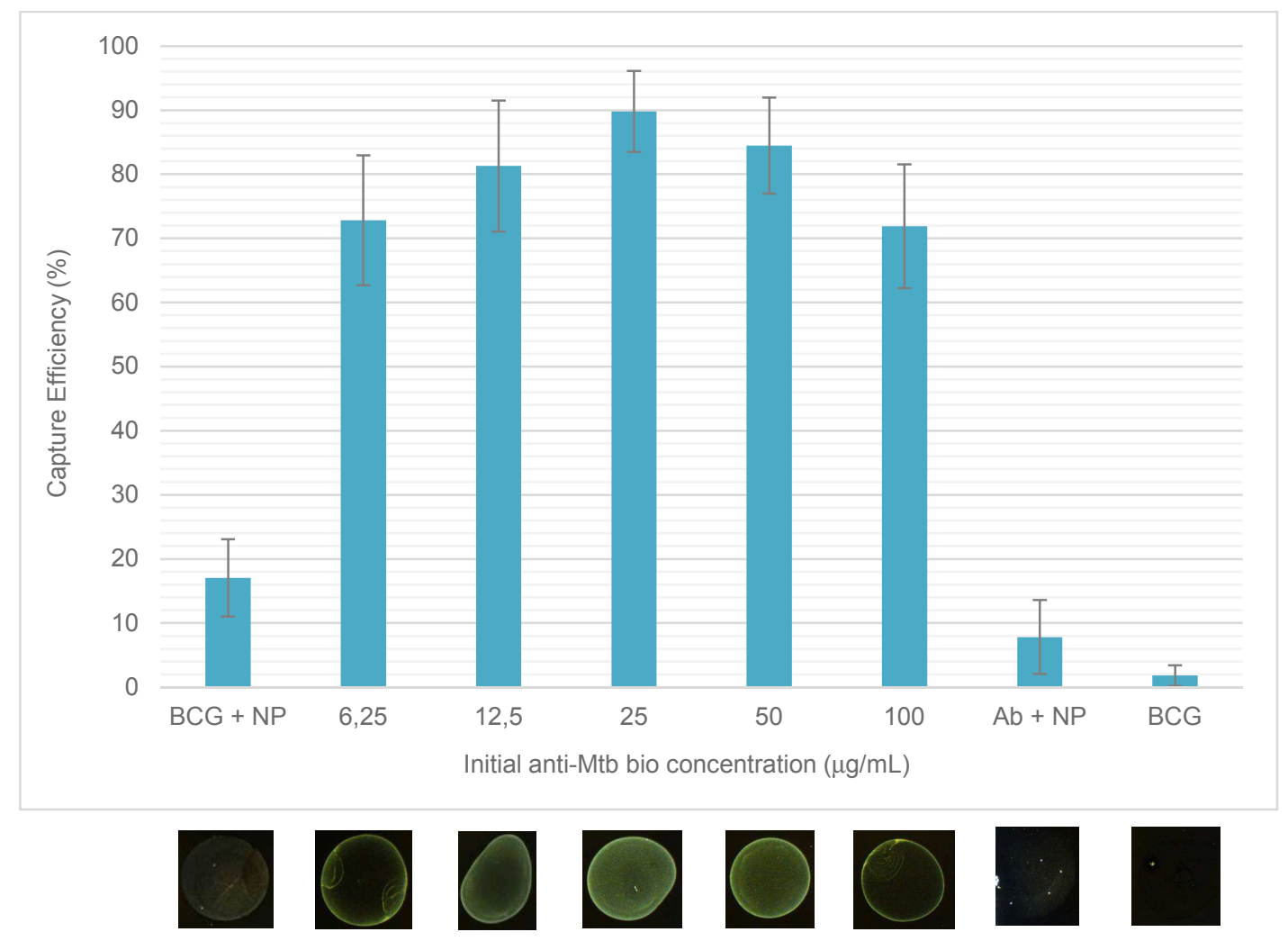

Figure 4. Surface coverage estimated by Image $J$, on the spot of the secondary anti-Mtb, depending on the initial concentration of anti-Mtb bio, and corresponding examples of the pictures on the gold substrates.

3.3 Optimization of the MNP@PAbs@BCG biomolecular recognition on the biochip. The examples of Fig. 5 show images of uncapsulated biochips with the 3 groups of 5 U-shaped sensors. The green sensors (reference) are not covered with gold, which means that were not chemically bound to the secondary PAbs for the immunoreaction. Therefore, the MNP (brown points) over the green sensors are the result of unspecific adsorption. Comparing the surface coverage obtained with the different solutions used for the ressuspension of MNP, it was found that water allowed higher 
binding, although there was an important degree of surface coverage in non-specific anti-Salm PAbs. PB reduced the binding of non-specific PAbs, maintaining a good coverage on specific antiMtb. Finally, it was observed that PBT disfavored PAbs immobilization both for specific and nonspecific PAbs.
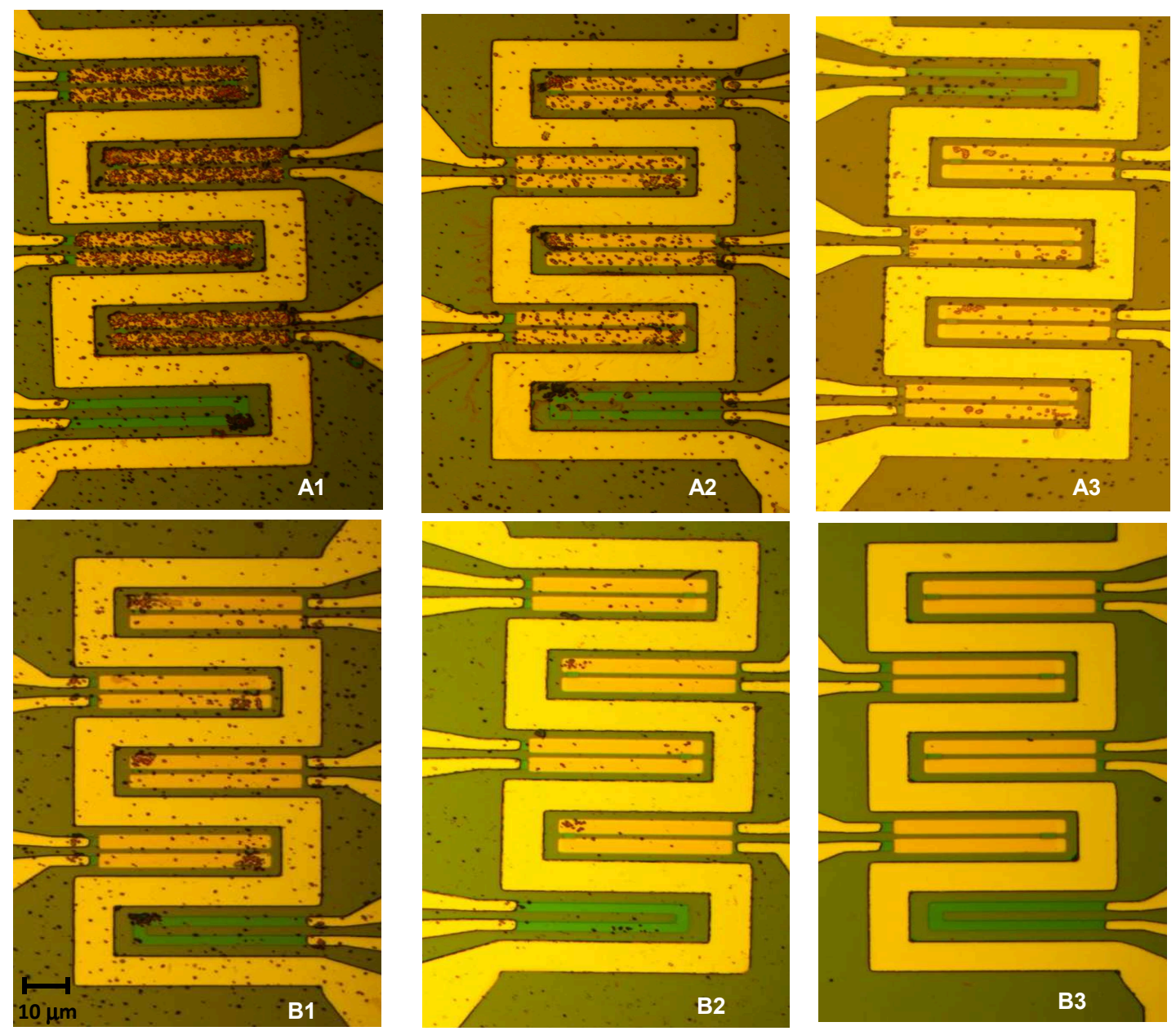

$A=$ anti-Mtb, $B=$ anti-Salm; $1=\mathrm{H}_{2} \mathrm{O}, 2=\mathrm{PB}, 3=\mathrm{PBT}$

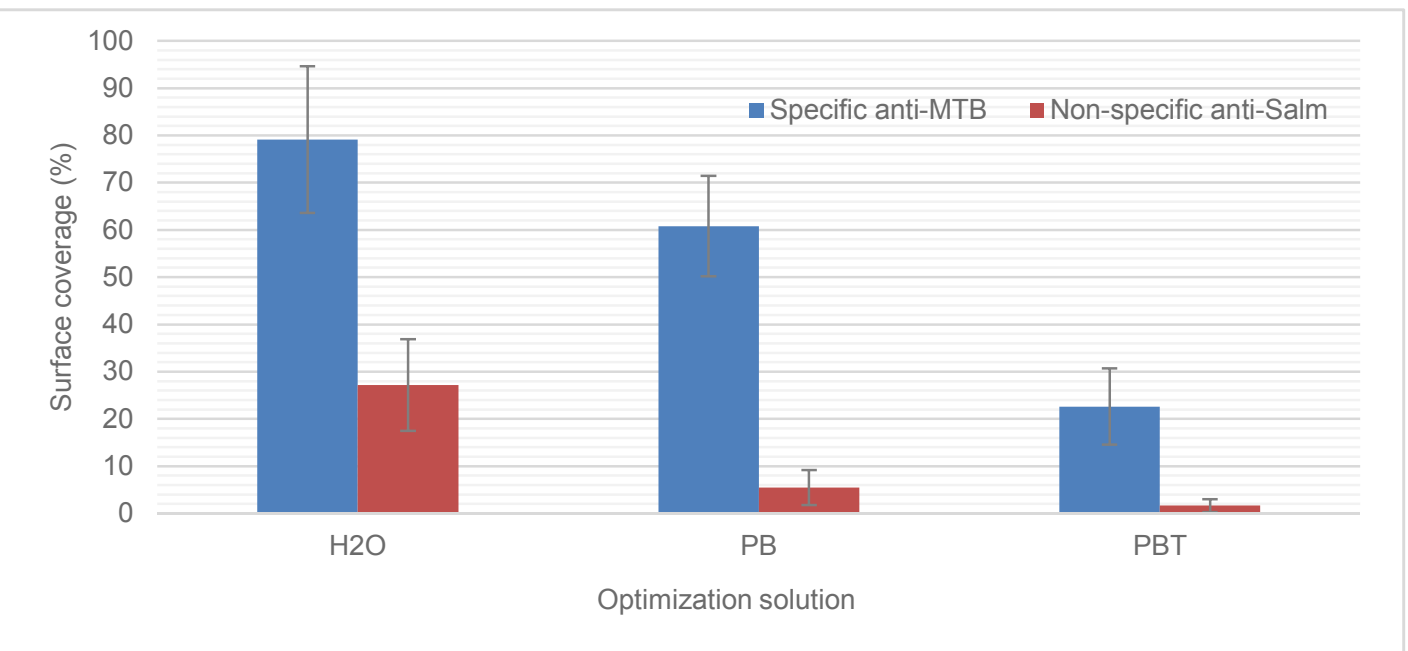

Figure 5. MNP surface coverage estimated by Image $J$ and corresponding examples of the pictures on the surface of uncapsulated biochips.

3.4 MR-biochip measurements. As presented in the examples of Fig. 6, the results on the MR sensors showed a clear difference in the voltage variation between specific and non-specific 
secondary PAbs, after the washing steps to remove unbound MNP (subtracting the voltage of the particles, $\mathrm{V}_{\text {particles, }}$, to the baseline signal). An average of $550 \mathrm{uV}$ against $50 \mathrm{uV}$ of $\Delta \mathrm{V}_{\text {binding }}$ signal (before normalizing to each sensor baseline signal), was obtained in sensors with the anti-Mtb positive control and the anti-Salm negative control, respectively. This voltage difference $(\Delta \mathrm{V})$, introduced by MNP in BCG cells immobilized by specific anti-Mtb, is directly proportional to the MNP number, and therefore to captured cells $[8,19]$.
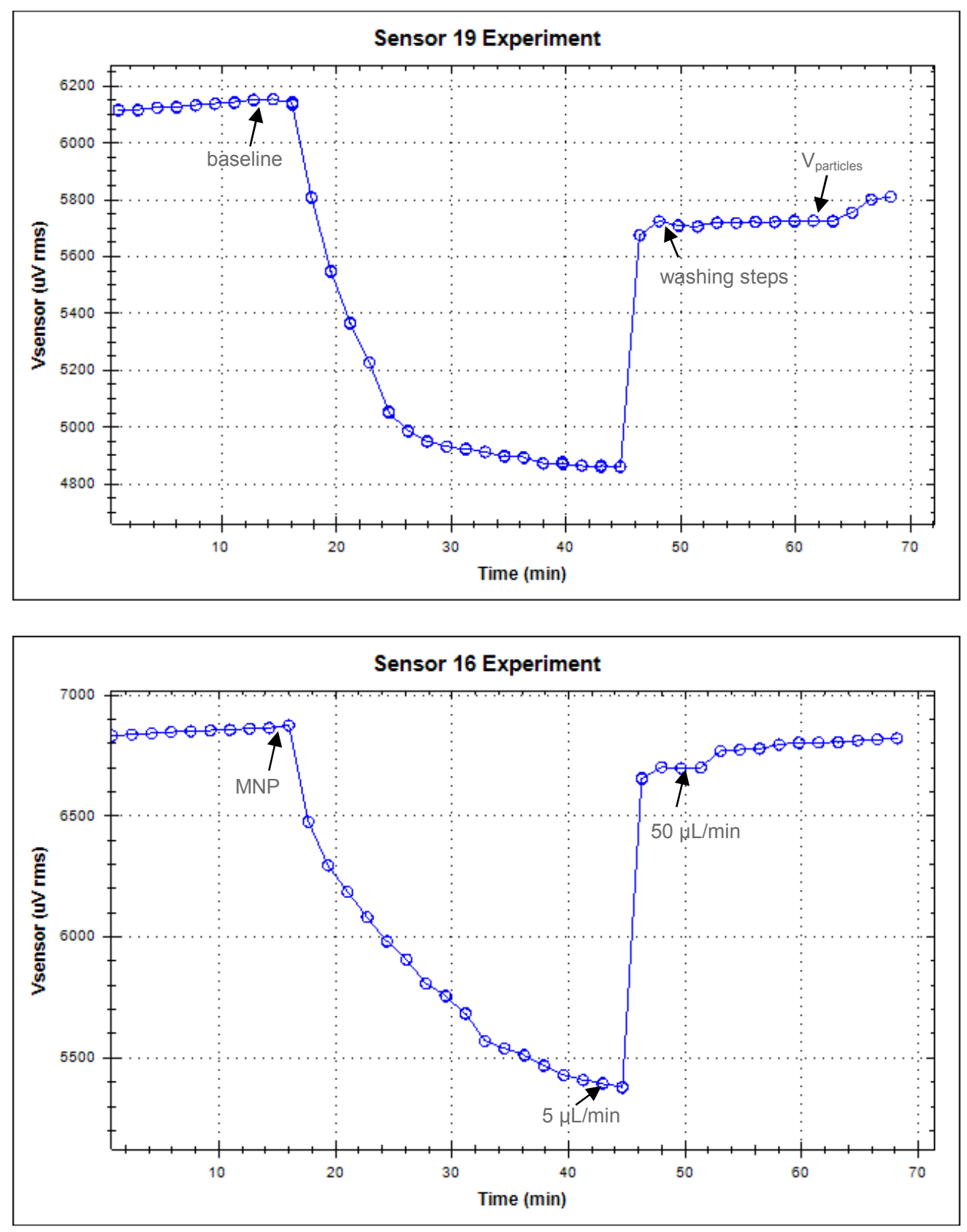

Figure 6. Comparison of the signal acquisition during the MR-biochip measurement between the anti-Mtb positive control (sensor 19) and the anti-Salm negative control (sensor 16) - example of two sensors.

At the end of the experiments, the signals from ten control sensors and ten active sensors were compared. The normalized average signals $\left(\Delta \mathrm{V} / \mathrm{V}_{\text {sensor }}\right)$ acquired for positive sensors covered with specific anti-Mtb or with negative sensors covered with nonspecific anti-Salm, after the washing of the unbound MNP on the chip surface at a flow rate of $50 \mu \mathrm{L} / \mathrm{min}$, presented a clear difference (about 9 times higher in the positive sensors, as shown in Fig. 7). These results were in agreement 
with the capturing of BCG cells by the specific secondary anti-Mtb, which remained attached to the sensor even after intensive washing, confirming the usefulness of this method as a biorecognition tool.

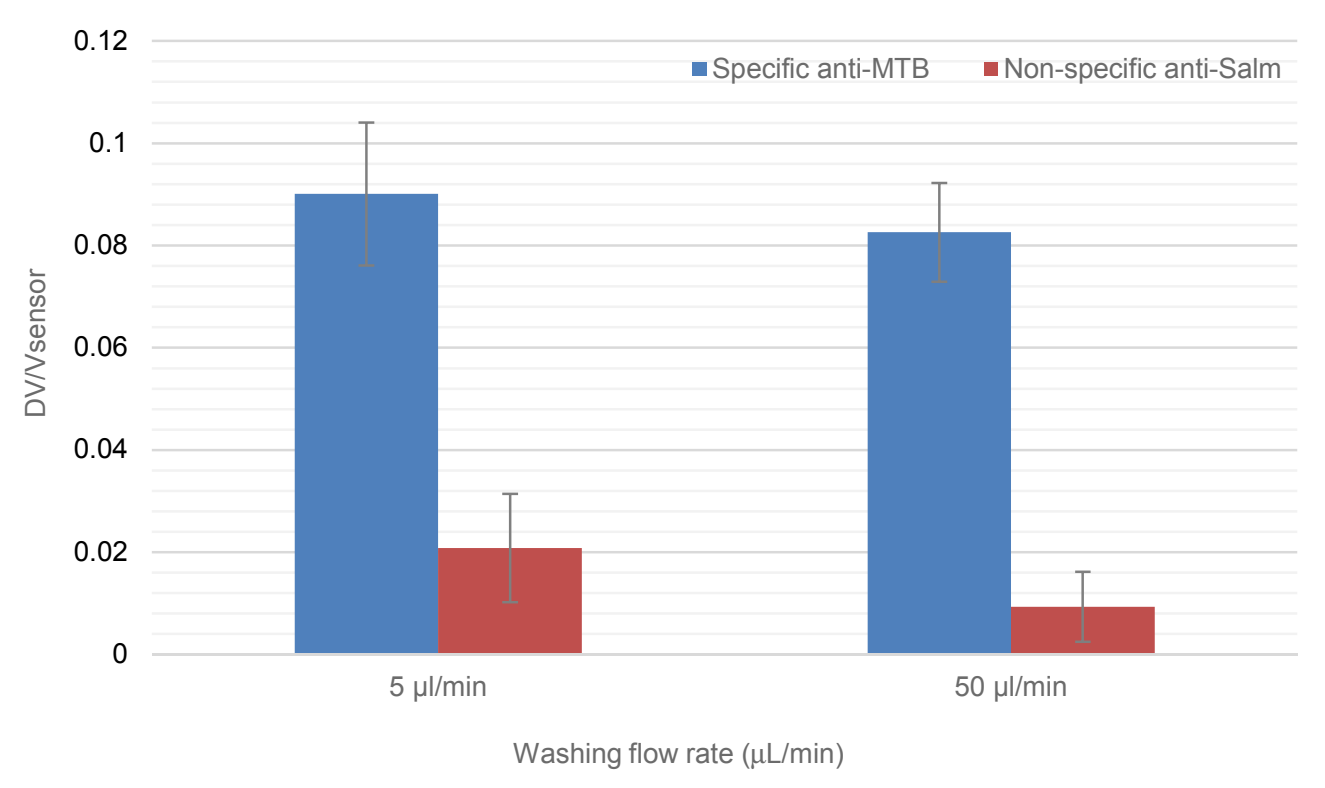

Figure 7. Comparison of the signal acquisition on the biochip between the anti-Mtb positive control and the anti-Salm negative control, at different washing flow rates.

\section{Conclusions}

The results confirmed that the BCG cells were successfully captured and magnetically labeled by the anti-Mtb bio MNP, as well as specifically detected by the sensors functionalized with anti-Mtb on the MR-biochip surface, by a sandwich-type immunoassay.

As an alternative to the gold-standard culture method, a new method towards a portable diagnostic platform for TB was investigated, with the goal of introducing a point-of-care, simple and fast way of detecting Mtb bacteria. Commercial MNP were used to label specific anti-Mtb bio and to capture BCG bacteria, and this system was introduced in the microfluidic chamber for direct detection. An MR biochip was used to demonstrate the capability of the method to capture the labeled probe at the surface of the sensor. The results showed a persistent signal from the MNP bound to the specific anti-Mtb Abs, which was significantly higher than the signal from negative control anti-Salm Abs, demonstrating the viability of the method.

Antigen detection platforms could constitute interesting alternatives to the existing TB diagnostic tests, since this method is simple, rapid and inexpensive, which makes it feasible in clinical setups of developing countries. Moreover, the herein proposed MR biochip platform can offer portability to the detection system and potentially increase the sensitivity of antibody-based diagnosis, by enhancing the amplification of the detection signal and reducing the bacterial amount required for the immunodiagnostic reaction.

In this experimental work, the specificity of a MR biochip platform was demonstrated in a laboratory scale with bulk solutions. It is our opinion that, with further research on designing and testing strategies for single-cell detection of untreated clinical samples (urine, sputum, serum), this method has the potential to become a standard diagnostic tool for the simple, fast and sensitive detection of $\mathrm{Mtb}$ and to act as a first line approach for laboratorial diagnosis of TB in point-of-care settings and poor resource countries. 


\section{References}

[1] World Health Organization, Global Tuberculosis Report 2014, Geneva (2014). Information on http://www.who.int/tb/publications

[2] World Health Organization, Global Tuberculosis Report 2014, Geneva (2013). Information on http://www.who.int/tb/publications

[3] F. Abebe, C. Holm-Hansen, H.G. Wiker, G. Bjune, Progress in serodiagnosis of Mycobacterium tuberculosis infection, Scandinavian Journal of Immunology. 66 (2007) 176-191.

[4] M.D. Perkins, J. Cunningham, Facing the Crisis: Improving the Diagnosis of Tuberculosis in the HIV Era, The Journal of Infectious Diseases. 196 (2007) S15-27.

[5] S.A. Martins, V.C. Martins, F.A. Cardoso, P.P. Freitas, L.P. Fonseca, Waterborne Pathogen Detection Using a Magnetoresistive Immuno-Chip, in: Springer Protocols Handbooks, Molecular Biological Technologies for Ocean Sensing, 2012, pp. 263-288.

[6] S.A. Martins, V.C. Martins, M.T.B. Frasco, A.G.B. Castro, P.P. Freitas, L.P. Fonseca, Magnetic labeling of waterborne pathogens towards a magnetoresistive-based biochip, Conference Proceeding of Ibersensor. 9-11 November, 2010, Lisbon, Portugal.

[7] J. Germano, V.C. Martins, F.A. Cardoso, T.M. Almeida, L. Sousa, P.P. Freitas, M.S. Piedade, A portable and autonomous magnetic detection platform for biosensing, Sensors (Basel). 9(6) (2009) 4119-37.

[8] E. Fernandes E, V.C. Martins, C. Nóbrega, C.M. Carvalho, F.A. Cardoso, S. Cardoso, J. Dias, D. Deng, L.D. Kluskens, P.P. Freitas, J. Azeredo, A bacteriophage detection tool for viability assessment of Salmonella cells, Biosensors and Bioelectronics. 52 (2014) 239-46.

[9] A. Cruz, A.G. Fraga, J.J. Fountain, J. Rangel-Moreno, E. Torrado, M. Saraiva, D.R. Pereira, T.D. Randall, J. Pedrosa, A.M. Cooper, A.G. Castro, Pathological role of interleukin 17 in mice subjected to repeated BCG vaccination after infection with Mycobacterium tuberculosis, The Journal of Experimental Medicine. ; 207(8) (2010) 1609-16.

[10] P. Bettencourt, D. Pires, N. Carmo, E. Anes, Application of confocal microscopy for quantification of intracellular mycobacteria macrophages, in: A. Méndez-Vilas, J. Díaz (Eds.), Microscopy: Science, Technology, Applications and Education, Badajoz: Formatex Research Center, 2010, 1, pp. 614-621.

[11]: M.M. Bradford, A rapid and sensitive method for the quantitation of microgram quantities of protein utilizing the principle of protein-dye binding, Analytical Biochemistry. 72 (1976) 248-54.

[12]: R. Treuer, S.E. Haydel, Acid-fast staining and Petroff-Hausser chamber counting of mycobacterial cells in liquid suspension, in: Current Protocols in Microbiology, 2011, Chapter 10, Unit 10A.6.

[13] V.C. Martins, F.A. Cardoso, J. Germano, S. Cardoso, L. Sousa, M. Piedade, P.P. Freitas, L.P. Fonseca, Femtomolar limit of detection with a magnetoresistive biochip, Biosensors and Bioelectronics. 24 (8) (2009) 2690-2695. 
[14] V.C. Martins, J. Germano, F.A. Cardoso, J. Loureiro, S. Cardoso, L. Sousa, M. Piedade, L.P. Fonseca, P.P. Freitas, Challenges and trends in the development of a magnetoresistive biochip portable platform, Journal of Magnetism and Magnetic Materials. 322 (9-12) (2010) 1655-1663.

[15] H. Zhang, P.S. Williams, M. Zborowski, J.J. Chalmers, Binding affinities/avidities of antibody-antigen interactions: quantification and scale-up implications, Biotechnology and Bioengineering. 95(5) (2006) 812-29.

[16] B. Byrne, E. Stack, N. Gilmartin, R. O’Kennedy, Antibody-Based Sensors: Principles, Problems and Potential for Detection of Pathogens and Associated Toxins, Sensors. 9 (2009) 44074445.

[17] V.C. Martins, F.A. Cardoso, P.P. Freitas, L.P. Fonseca, Picomolar detection limit on a magnetoresistive biochip after optimization of a thiol-gold based surface chemistry, Journal of Nanoscience and Nanotechnology. 10 (9) (2010) 5994-6002.

[18] B. Masereel, M. Dinguizli, C. Bouzin, N. Moniotte, O. Feron, B. Galle, T.V. Borght, C. Michiels, S. Lucas, Antibody immobilization on gold nanoparticles coated layer-by-layer with polyelectrolytes, Journal of Nanoparticle Research. 13(4) (2011) 1573-1580.

[19] F.A. Cardoso, J. Germano, R. Ferreira, S. Cardoso, V.C. Martins, P.P. Freitas, M.S. Piedade, L. Sousa, Detection of $130 \mathrm{~nm}$ magnetic particles by a portable electronic platform using spin valve and magnetic tunnel junction sensors, Journal of Applied Physics. 103 (2008) 07A310. 
Journal of Nano Research Vol. 34

10.4028/www.scientific.net/JNanoR.34

Detecting Antibody-Labeled BCG MNPs Using a Magnetoresistive Biosensor and Magnetic Labeling Technique

10.4028/www.scientific.net/JNanoR.34.49 